\section{Prmt5 is essential for early mouse development and acts in the cytoplasm to maintain ES cell pluripotency}

\author{
Wee-Wei Tee, ${ }^{1}$ Mercedes Pardo, ${ }^{2}$ \\ Thorold W. Theunissen, ${ }^{1} \mathrm{Lu} \mathrm{Yu}{ }^{2}$ \\ Jyoti S. Choudhary, ${ }^{2}$ Petra Hajkova, ${ }^{3}$ \\ and M. Azim Surani ${ }^{1,4}$ \\ ${ }^{1}$ Wellcome Trust, Cancer Research UK, Gurdon Institute \\ of Cancer and Developmental Biology, University of Cambridge, \\ Cambridge CB2 1QN, United Kingdom; ${ }^{2}$ Proteomic Mass \\ Spectrometry, Wellcome Trust Sanger Institute, Hinxton, \\ Cambridge CB10 1SA, United Kingdom; ${ }^{3}$ MRC Clinical \\ Sciences Centre, Imperial College, London W12 0NN, \\ United Kingdom
}

Prmt5, an arginine methyltransferase, has multiple roles in germ cells, and possibly in pluripotency. Here we show that loss of Prmt5 function is early embryonic-lethal due to the abrogation of pluripotent cells in blastocysts. Prmt5 is also up-regulated in the cytoplasm during the derivation of embryonic stem (ES) cells together with Stat3, where they persist to maintain pluripotency. Prmt5 in association with Mep50 methylates cytosolic histone H2A (H2AR3me2s) to repress differentiation genes in ES cells. Loss of Prmt5 or Mep50 results in derepression of differentiation genes, indicating the significance of the Prmt5/Mep50 complex for pluripotency, which may occur in conjunction with the leukemia inhibitory factor (LIF)/Stat3 pathway.

Supplemental material is available for this article.

Received August 21, 2010; revised version accepted November 1, 2010.

Prmt5, an arginine methyltransferase, has multiple cytoplasmic and nuclear substrates, including histone H2A/ H4 (H2A/H4R3me2s) (Bedford and Clarke 2009). We showed previously that Prmt5 has a role in the establishment and propagation of unipotent primordial germ cells (PGCs) from embryonic day 7.5 (E7.5) to E11.5, where it cooperates with Blimp1/Prdm1 (Ancelin et al. 2006), a key regulator of PGC specification. During reprogramming of E8.5 unipotent PGCs back to pluripotent embryonic germ (EG) cells in vitro, Blimp1/Prdm1 is rapidly down-regulated, but Prmt5 translocates to the cytoplasm, where it persists thereafter, suggesting that cytosolic Prmt5 might have another distinct role in pluripotency (Durcova-Hills et al. 2008). Indeed, cytosolic Prmt5 is also

[Keywords: Embryonic stem cells; pluripotency; epigenetics; arginine methyltransferase; H2A]

${ }^{4}$ Corresponding author.

E-MAIL a.surani@gurdon.cam.ac.uk, FAX 44-1223-334089.

Article is online at http://www.genesdev.org/cgi/doi/10.1101/gad.606110. detected in embryonic stem (ES) cells derived from blastocysts (see later).

Pluripotency is established during preimplantation development, where a number of factors, including epigenetic regulators, play a critical role (Surani et al. 2007). Pluripotent cells are established in the inner cell mass (ICM) of blastocyst at E3.5, together with the outer trophectoderm (TE) cells. The pluripotent cells can be propagated indefinitely in vitro, which requires an interplay between transcription factors, epigenetic regulators, and signal transduction pathways, which ensures repression of differentiation (Niwa 2007).

Here we investigate the role of Prmt5 in mouse development, when pluripotent cells are established and can be propagated as ES cells. Prmt5 is essential for early development and, as a cytosolic factor, it is important for the derivation and maintenance of pluripotent ES cells, partly in association with Mep50, to direct methylation of predeposited histone $\mathrm{H} 2 \mathrm{~A}$.

\section{Results and Discussion}

Dynamic expression of Prmt5 in early embryos and during ES cell derivation

First, we found that Prmt5 is maternally inherited in the oocyte cytoplasm and is excluded from the metaphase chromosomes. It remained cytoplasmic in the zygote and was excluded from the pronuclei (PN) (Fig. 1A). During the eight-cell to 16-cell transition, where totipotency is lost, Prmt5 translocated into the nucleus; this event coincides with the initiation of the first cell fate decision toward the establishment of the outer TE and the ICM, which contains the founder pluripotent cells (Fig. 1A). In E3.5 blastocysts, Prmt5 is nuclear in both the inner and outer cells, and, at E4.5, it persists in the nuclei of differentiated TE cells but is down-regulated transiently in the pluripotent ICM epiblast cells. At E6.5, we again detected Prmt5 in the pluripotent epiblast cells of the post-implantation embryo, but predominantly in the cytoplasm (Fig. 1A). These observations indicate the dynamic nature of Prmt5 localization in the nucleus and cytoplasm in early development.

The transient loss of Prmt5 in the ICM epiblast is noteworthy considering that there are high levels of Prmt5 in ES cells. We therefore investigated E3.5 blastocysts cultured in the presence of the cytokine leukemia inhibitory factor (LIF) and fetal calf serum (FCS) to induce ICM outgrowths from which ES cells are derived. We found transient loss of Prmt5 in the epiblast over 24-h culture (Fig. 1B), but it reappeared predominantly in the cytoplasm of all ICM outgrowth cells, including Oct4positive pluripotent cells (Fig. 1B), which is reminiscent of cytosolic expression in the E6.5 post-implantation epiblast. Therefore, the re-expression of Prmt5 may be coupled with development and proliferation of the epiblast in vivo and in vitro. Notably, the down-regulation of Prmt5 in the epiblast cells must have occurred by a posttranscriptional mechanism, since Prmt5 transcription persists throughout this period (Fig. 1C). However, the underlying mechanism is currently unknown. This regulated and precipitant loss of predominantly nuclear-localized Prmt5, and its subsequent reappearance in the cytoplasm, signify an important developmental transition, which is 
A

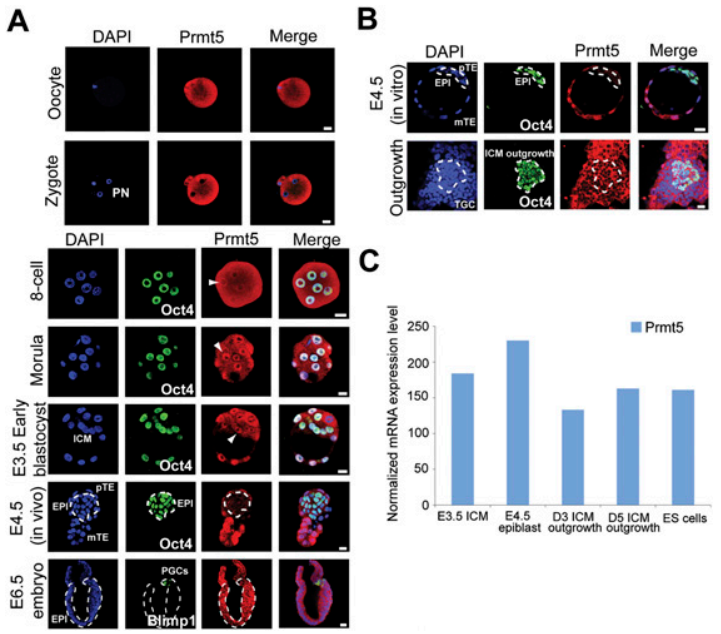

Figure 1. Dynamic expression of Prmt5 in early embryos and ES cell derivation. (A) Expression of Prmt5 (red) at various stages of early development. Oct4-positive cells as well as Blimp1-GFPpositive founder PGCs in E6.5 epiblasts are denoted in green. Nuclei were counterstained with DAPI (blue). (EPI) Epiblast; (pTE) polar TE; (mTE) mural TE. Bar, $20 \mu \mathrm{m}$. (B) Prmt5 (red) is up-regulated in the ICM outgrowth during ES cell derivation in vitro. Oct4 (green) marks pluripotent cells. (TGC) Trophoblast giant cells. (C) Prmt5 mRNA expression remains unchanged in Oct4-positive cells in the course of ES derivation, based on single-cell RNA-Seq analysis (Tang et al. 2010).

reminiscent of the translocation of Prmt5 from the nucleus to cytoplasm during reprogramming of unipotent PGCs to pluripotent EG cells in vitro (Durcova-Hills et al. 2008).

\section{Prmt5 is required for embryonic development} and the derivation of ES cells

Next, we asked if the early expression of Prmt5 is essential for the development and derivation of ES cells, using mutant mice generated from $\mathrm{Prm}^{+/-}$ES cells containing a gene trap vector inserted between exons 6 and 7 that disrupts most of the methyltransferase domain (Supplemental Fig. 1). Whereas Prmt5 $5^{+/-}$mice were normal and fertile, we found no viable homozygous offspring (Supplemental Table 1), and, furthermore, we confirmed early embryonic lethality, where mutant embryos lacked discernable embryonic structures at E6.5 (Supplemental Fig. 1).

Next, we examined Prmt5 $5^{-/-}$E3.5 mutant blastocysts, which we recovered at a normal Mendelian ratio, and which were morphologically indistinguishable from wildtype embryos (Supplemental Fig. 1). Mutant blastocysts cultured in vitro in the context of ES cell derivation hatched from the zona pellucida and attached, resulting in the formation of trophoblast giant cells, but the ICM failed to grow (Fig. 2A). Notably, no Prmt5-null ES cells could be obtained, indicating that the up-regulation and translocation of Prmt5 to the cytoplasm is as critical for the derivation of ES cells as it is for embryonic development in vivo.

\section{Prmt5 is cytoplasmic in ES cells and essential} for pluripotency

We found that Prmt5 is highly expressed in ES cells and is present almost exclusively in the cytoplasm (Fig. 2B), which further strengthens the association between cytosolic Prmt5 and pluripotency. In contrast, Prmt5 is present in the nucleus of TE stem cells in vitro (Fig. 2B) - which are developmentally restricted to forming the extraembryonic tissues only-and in differentiating ES cells that are Oct4-negative (Fig. 2B). This suggests that the differential nuclear or cytoplasmic localization may reflect diverse roles of Prmt5 in pluripotency and cell fate determination.

Next, we used RNAi to deplete Prmt5 in ES cells, which led to pronounced morphological changes and loss of alkaline phosphatase staining, indicating a loss of pluripotency (Fig. 3A). Notably, loss of Prmt5 also resulted in down-regulation of key pluripotency genes, including Oct4, Nanog, and Rex1, together with a reduction in Oct4 and Nanog proteins (Fig. 3A). Concomitantly, we detected up-regulation of several differentiation genes, including Fgf5, Gata4, Gata6, and HoxD9, as shown by quantitative PCR (qPCR) (Fig. 3B). Furthermore, global gene expression analysis following Prmt5 knockdown confirmed that numerous other ES-associated genes were significantly down-regulated, while developmentally regulated genes were up-regulated (Fig. 3C; Supplemental Table 2). Gene ontology (GO) analysis confirmed down-regulation of "stem cell maintenance" genes, and up-regulation of "embryonic development" genes (Supplemental Table 2), which demonstrates that cytoplasmic Prmt5 is crucial for the maintenance of pluripotency in ES cells. We pursued this further for mechanistic insights on the role of Prmt5 in ES cells.

\section{Predeposited histone H2A is a substrate of cytoplasmic Prmt5 in ES cells}

Epigenetic regulators play a significant role in the repression of differentiation genes in ES cells (Boyer et al.

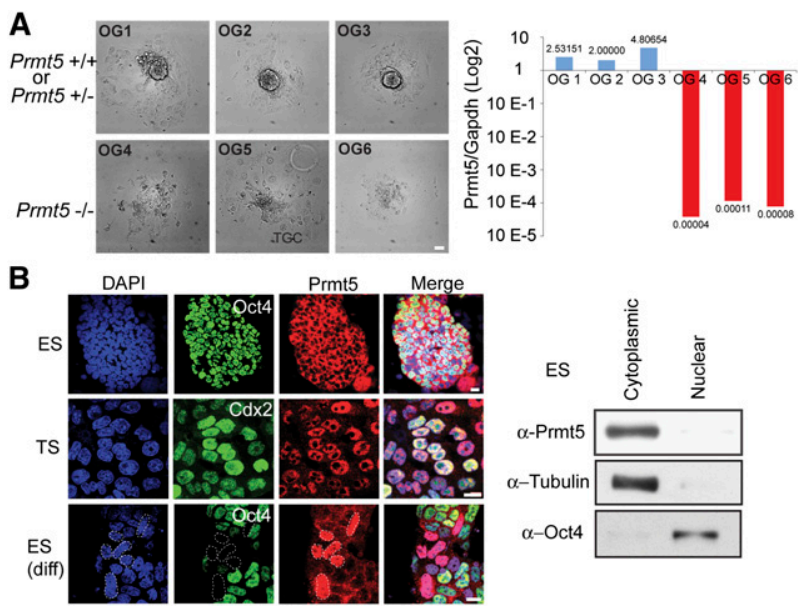

Figure 2. Effects of Prmt5 loss of function and detection of cytoplasmic Prmt5 in ES cells. (A) A representative display of six blastocyst outgrowths (OG) from a Prmt5 $5^{+-}$intercross. Outgrowths 4-6 showed impaired ICM proliferation, and were verified as Prmt $5^{-1-}$ by qPCR. Levels of Prmt 5 transcript were normalized against Gapdh, and results are shown on a $\log 2$ scale. Bar, $20 \mu \mathrm{m} .(B)$ Immunostainings of Prmt5 (red) in pluripotent ES, TS, and differentiated ES cells. Oct4 and Cdx2 are in green. Nuclei were counterstained with DAPI (blue). (Right panel) Western blot of cytoplasmic and nuclear fractions from ES cells confirms cytoplasmic location of Prmt5. Purity of the preparations was confirmed by Oct4 (nuclear) and Tubulin (cytoplasmic) expression. Bar, $20 \mu \mathrm{m}$. 
A
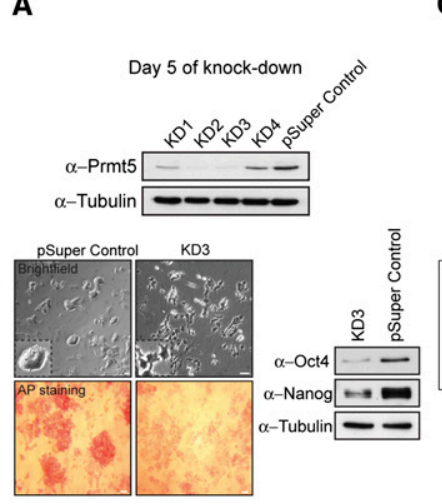

C

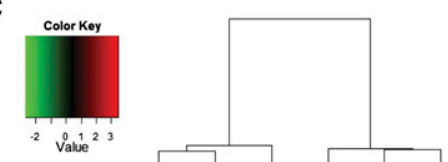

B

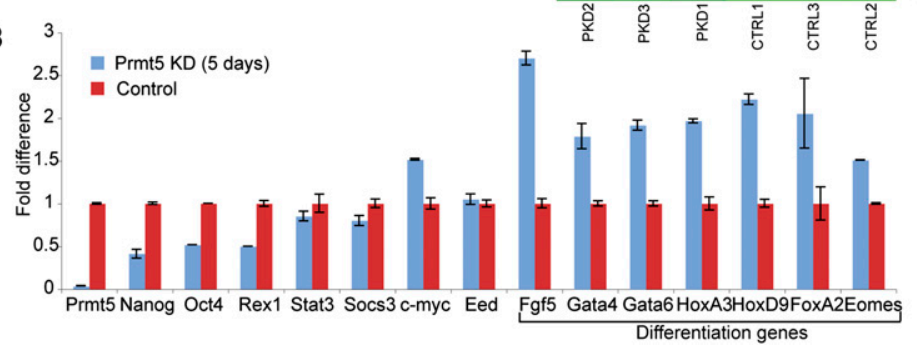

Figure 3. Prmt5 is required for ES cell pluripotency. $(A)$ Western blots depicting efficiencies of Prmt5 shRNA knockdown constructs (KD 1-4) compared with control (pSuper Control). Note phenotypic changes, diminution of Oct4 and Nanog, and alkaline phosphatase staining in Prmt5-depleted cells $5 \mathrm{~d}$ after knockdown. Tubulin was used as loading control. Bar, $30 \mu \mathrm{m}$. (B) qPCR analysis of gene expression in Prmt5 knockdown cells harvested $5 \mathrm{~d}$ after knockdown. Levels of transcripts were normalized relative to control shRNA transfection. Error bars indicate standard deviations of three technical replicates. $(C)$ Microarray heat map depicting expression changes of selected ES-associated genes as well as developmental genes after Prmt5 knockdown $(P<0.01)$. Biological triplicates were used. (PKD) Prmt5 knockdown; (CTRL) control knockdown.

2006; Bilodeau et al. 2009; Yeap et al. 2009; Yuan et al. 2009), usually in the context of chromatin. Prmt5 can methylate histones $\mathrm{H} 2 \mathrm{~A} / \mathrm{H} 4$ to generate $\mathrm{H} 2 \mathrm{AR} 3 \mathrm{me} 2 \mathrm{~s} / \mathrm{H} 4 \mathrm{R} 3 \mathrm{me} 2 \mathrm{~s}$ modifications that are associated with gene repression (Pollack et al. 1999; Ancelin et al. 2006). However, since Prmt5 is cytoplasmic in ES cells, we first checked for enzymatic activity toward histones. We found that Prmt5 immunoprecipitated from ES cells could methylate histones $\mathrm{H} 2 \mathrm{~A}$ and $\mathrm{H} 4$ in vitro (Fig. 4A). We then asked if cytoplasmic Prmt5 in ES cells in vivo has a role in histone methylation, by investigating the levels of H2AR3me2s- and H4R3me2srepressive modifications after Prmt5 knockdown using the available antibody that primarily recognizes H4R3me2s modification, but that also cross-reacts with methylated histone H2A (Ancelin et al. 2006; Shechter et al. 2009). Interestingly, when we examined histones from ES cells depleted of Prmt5 using acid extraction, there was no significant loss of H4R3me2s modification, but we observed a dramatic and selective loss of H2AR3me2s modification (Fig. 4B). Given that Prmt5 is predominantly cytoplasmic and is not associated directly with the chromatin in ES cells (Supplemental Fig. 2), this observation hints at a possibility that predeposited histone $\mathrm{H} 2 \mathrm{~A}$ might be a key substrate of Prmt5 in ES cells in vivo.

To pursue this observation further, we examined the nuclear fraction of ES cells, and, as expected, arginine methylation of both histone $\mathrm{H} 2 \mathrm{~A}$ and $\mathrm{H} 4$ was detected. In contrast, in the cytoplasmic fraction, we found that only $\mathrm{H} 2 \mathrm{~A}$ was predominantly methylated (Fig. 4B), which further supports the idea that Prmt5 might be important for the selective methylation of cytoplasmic predeposited H2A as one of its key targets. We obtained additional evidence in Prmt5 knockdown ES cells examined for up to $5 \mathrm{~d}$, which showed rapid loss of H2AR3me2s in the cytosolic fraction at day 3 , coincidentally with the down-regulation of Prmt5, while the nuclear H2AR3me2s levels changed in a more protracted fashion by $5 \mathrm{~d}$ (Fig. 4C). The kinetics of the loss of H2ARme2s in Prmt5-depleted ES cells-first in the cytoplasm and then nuclear-lends credence to the idea that Prmt5 might methylate predeposited $\mathrm{H} 2 \mathrm{~A}$ histones prior to their incorporation into the chromatin.

To independently assess if methylated H2A histones are involved in the repression of some differentiation genes, we overexpressed methylation-deficient mutant $\mathrm{H} 2 \mathrm{~A}\left(\mathrm{H} 2 \mathrm{~A}^{\mathrm{R} 3 \mathrm{~A}}\right)$ histones in ES cells, which were readily incorporated into chromatin (Supplemental Fig. 3) and resulted in a significant up-regulation of several of the differentiation genes (Fig. 4D). Many of these genes were the same as those that were up-regulated following the loss of Prmt5 in ES cells. Although some genes were not affected, this might be because of differences in the extent to which $\mathrm{H} 2 \mathrm{~A}^{\mathrm{R} 3 \mathrm{~A}}$ are incorporated into various promoters. Notably, expression of pluripotency genes was unaffected by $\mathrm{H} 2 \mathrm{~A}^{\mathrm{R} 3 \mathrm{~A}}$, which rules out nonspecific effects of $\mathrm{H} 2 \mathrm{~A}^{\mathrm{R} 3 \mathrm{~A}}$ 'overexpression in ES cells and points to one particular role of Prmt5 in repression of differentiation genes through methylation of histone $\mathrm{H} 2 \mathrm{~A}$, among others. Importantly, introduction of methylation-deficient mutant histones $\mathrm{H} 4$ $\left(\mathrm{H} 4^{\mathrm{R} 3 \mathrm{~A}}\right)$ had no effect (Fig. 4D), which is consistent with a lack of effect on H4 methylation following Prmt5 knockdown. These observations support the role of Prmt5mediated arginine methylation in the maintenance of pluripotent ES cells by repression of differentiation-specific genes, in part through methylation of histone H2A.

\section{The role of Mep50-Prmt5 in methylation of predeposited H2A in ES cells}

To obtain further support for our observations on methylation of predeposited H2A, we examined Mep50 (also called Wdr77), a WD repeat-containing protein that we detected as an interactor of Prmt5 in ES cells (Supplemental Fig. 4A). Mep50 is a key enzymatic cofactor of Prmt5 (Meister et al. 2001; Friesen et al. 2002) and specifically directs Prmt5 toward nonnucleosomal histone H2A but not H4 (Furuno et al. 2006). Notably, Mep50 is significantly down-regulated during differentiation of ES cells, together with H2AR3me2s modification (Fig. 5A), consistent with a likely role of Prmt5/Mep50-mediated H2AR3me2s methylation in maintaining ES cell pluripotency. Importantly, Mep50, like Prmt5, is predominantly cytoplasmic in ES cells, and, furthermore, we confirmed Mep50-Prmt5 interaction by coimmunoprecipitation. Moreover, immunofluorescence studies clearly showed colocalization of Prmt5 and Mep50 in the cytoplasm (Fig. 5B).

We checked the response of ES cells following Mep50 knockdown, and found that this resulted in the selective 
A

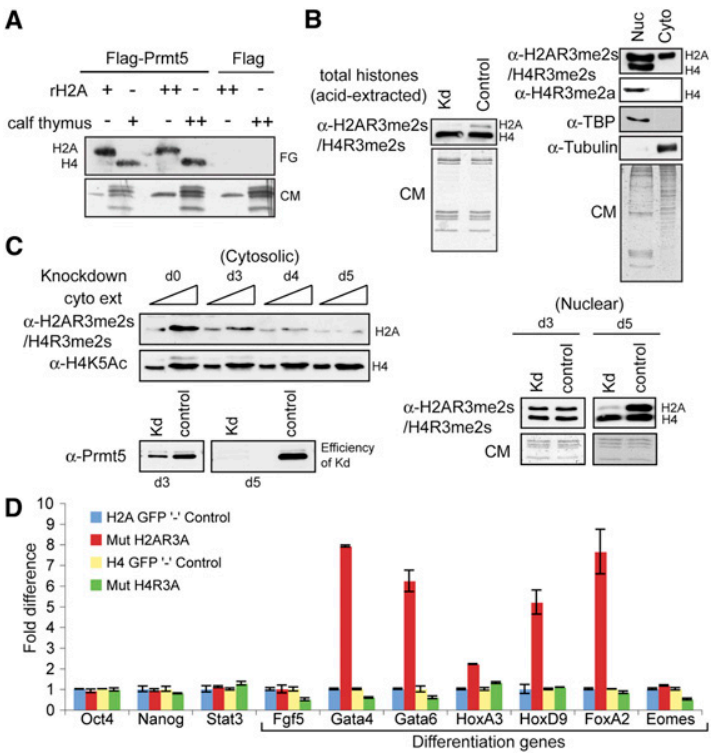

Figure 4. Prmt5 methylates predeposited histone H2A. (A) In vitro methyltransferase assay using Flag-Prmt5 immunoprecipitated from ES cells and incubated with histone substrates (recombinant H2A, $\mathrm{rH} 2 \mathrm{~A}$, or calf thymus histone preparations) and radioactive ${ }^{3} \mathrm{H}$-SAM. Fluorograph (FG) and Coomassie (CM) blots are shown. (B) Western blot showing loss of H2AR3me2s in Prmt5-depleted cells. (Left panel) Histones were isolated using acid extraction at day 5 of knockdown. ES cell fractions show H2AR3me2s/H4R3me2s in nuclear fraction but only H2AR3me2s in the cytoplasm (long Western blot exposure was performed). Purity of the preparations was confirmed by TBP (TATA-binding protein) (nuclear) and Tubulin (cytoplasmic) expression. (Right panel) H4R3me2a (asymmetric H4 dimethylation). (C) Time-course experiment showing loss of cytosolic H2AR3me2s upon depletion of Prmt 5 in ES cells between 0 and $5 \mathrm{~d}$. H4K5 acetylation (H4K5Ac) was used as a marker for cytosolic $\mathrm{H} 4$ and also served as loading control. Cytosolic H2AR3me2s was down-regulated first on day $3(\mathrm{~d} 3)$, concomitantly with the loss of Prmt5. Nuclear H2AR3me2s was subsequently down-regulated on day 5 (d5). (D) qPCR analysis of gene expression changes upon overexpression of methylation-deficient $\mathrm{H} 2 \mathrm{~A}$ and $\mathrm{H} 4$ histones. Mutant histones (containing IRES-GFP) were transfected into ES cells, and both "GFP" $\mathrm{GP}^{+}$" $\mathrm{GFP}^{-}$" (control) cells were isolated by FACS and subjected to qPCR. Error bars represent the standard deviations of three technical replicates.

loss of H2AR3me2s signal (Fig. 5C). This observation reinforced the role of Prmt5/Mep50 in the methylation of predeposited H2A. Importantly, loss of Mep50 also induced ES cell differentiation (Fig. 5C), resulting in an upregulation of differentiation genes, as we observed following Prmt5 knockdown and $\mathrm{H} 2 \mathrm{~A}^{\mathrm{R} 3 \mathrm{~A}}$ overexpression (Fig. 5D). However, as with $\mathrm{H} 2 \mathrm{~A}^{\mathrm{R} 3 \mathrm{~A}}$ overexpression, pluripotency genes were less affected upon loss of Mep50 (Fig. 5D). This is in contrast to the pronounced downregulation of pluripotency genes in response to Prmt5 knockdown. These observations led us to conclude that, whereas Prmt5/Mep50-mediated H2A methylation may play an instructive role in ensuring repression of differentiation genes, Prmt5 itself has additional independent roles in maintaining ES cell pluripotency.

\section{Prmt5-mediated repression of ES cell differentiation} may involve Stat3

Thus far, we focused on one aspect of Prmt5 function in ES cells on the Mep50-mediated methylation of prede- posited H2A histones that may contribute to the repression of differentiation genes. However, Prmt5 can also methylate nonhistone substrates, which may also regulate pluripotency. We set off to identify the interactors of cytoplasmic Prmt5 in an objective manner by performing mass spectrometry on the Prmt5 immunocomplexes using ES cells stably expressing Prmt5-Flag (Supplemental Fig. 4A). Mass spectrometry analysis identified both the known and novel interactors of Prmt5, including Mep50 (as described previously) and Gemin5 (Gubitz et al. 2002). Notably, we also detected Stat3, a key pluripotency transcription factor downstream from the LIF signaling pathway (Supplemental Fig. 4A).

LIF/Stat3 signaling plays a key role in the derivation and maintenance of pluripotent ES cells through transcriptional regulation (Niwa et al. 1998; Takeda et al. 1997; Matsuda et al. 1999|. Furthermore, Stat3 can bind to both pluripotency and differentiation genes in ES cells, suggesting that it has a role in both gene activation and repression (Kidder et al. 2008). To determine if Prmt5 might act in conjunction with Stat3, we first examined the expression of Stat3, and noted some similarities with Prmt5 in the early embryos. Like Prmt5, Stat 3 is transiently down-regulated in the epiblast of E4.5 blastocysts, followed by up-regulation in the ICM outgrowth during ES cell derivation (Supplemental Fig. 4B). Additionally, like the Prmt5-null embryos, Stat3 loss of function is early embryonic-lethal (Takeda et al. 1997).

We confirmed Prmt5-Stat3 interaction by coimmunoprecipitation in ES cells, and also found that the activated

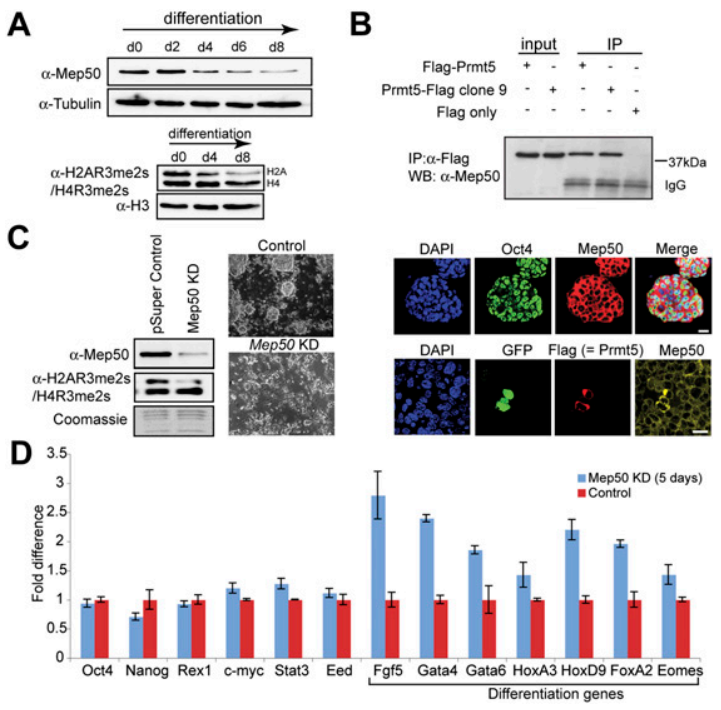

Figure 5. Prmt5/Mep50-mediated H2AR3me2s is important for pluripotency. $(A)$ Down-regulation of Mep50 during differentiation of ES cells. Loss of Mep50 at day 4 (d4) of differentiation was accompanied by a specific decrease in H2AR3me2s. (B) Prmt5 and Mep50 interact in ES cells. Immunoprecipitations using ES cells either transiently transfected with Flag-Prmt5 or stably expressing Prmt5-Flag (clone 9) and blotted for endogenous Mep50. Immunostainings showing colocalization of Mep50 with Flag-Prmt5 in the cytoplasm of ES cells. Transfected cells are denoted by GFP staining. Nuclei were counterstained with DAPI (blue). Bar, $20 \mu \mathrm{m} .(C)$ Mep50 knockdown (5 d) leads to loss of H2AR3me2s and results in ES cell differentiation. Bar, $30 \mu \mathrm{m}$. (D) qPCR analysis of gene expression upon Mep50 knockdown (5 d) in ES cells. Levels of transcripts were normalized with respect to control shRNA transfection. Error bars indicate standard deviations of three technical replicates. 
form of Stat3 (phosphorylated on Y705; pStat3[Y705]) exists in the Prmt5 immunocomplex, which is also supported by their coelution in gel filtration. However, phosphorylation of Y705 on Stat3 is not obligatory for the Stat3-Prmt5 interaction (Supplemental Fig. 4C). In vitro methylation assay also revealed no detectable methylation of Stat3, suggesting that it is not a substrate of Prmt5, at least in vitro (Supplemental Fig. 4D; data not shown). Furthermore, activation of the LIF/Stat3 pathway itself was not perturbed in Prmt5-depleted ES cells, since expression of Stat3-activated targets, such as Socs3 and Stat3 itself, was not significantly affected (Fig. 3A), and the levels of pStat3(Y705) were similar in both the Prmt5 knockdown and control ES cells (Supplemental Fig. 4E). Therefore, Prmt5 does not appear to regulate Stat3-dependent gene activation. In contrast, depletion of Prmt5 in ES cells caused up-regulation of differentiation genes, including Fgf5, Gata6, Lhx1, and FoxA2, and several Hox genes such as $\operatorname{Hox} A 3, \operatorname{Hox} A 7$, and $\operatorname{Hox} D 9$ (Fig. 3B,C). Notably, all of these are known direct repressed targets of Stat3 (Kidder et al. 2008), which suggests that the effect of loss of Prmt5 might be predominantly on the Stat3-bound repressed targets.

Although our observations suggest that Prmt5-Stat3 association might function to selectively repress differentiation genes in ES cells, how this occurs in conjunction with the methylation of predeposited $\mathrm{H} 2 \mathrm{~A}$ remains to be elucidated. However, we note an interaction between Stat3 and Nap1 (Supplemental Fig. 5), a histone chaperone for H2A-H2B dimers (Mosammaparast et al. 2002), which might provide a possible route for the incorporation of H2AR3me2s into the chromatin. Notably, a mechanism for signal-based targeted deposition of histones was demonstrated previously for histones H3$\mathrm{H} 4$, which involves chaperone Asf1 in conjunction with the DNA-binding factor $\mathrm{Su}(\mathrm{H})$, for the repression of Notch target genes (Goodfellow et al. 2007). Indeed, more recently, distinct DNA-binding factors were found to be responsible for targeted deposition of histone variant $\mathrm{H} 3.3$ in ES cells on both active and repressed genes in a DNA replication-independent manner (Goldberg et al. 2010). It is therefore conceivable that deposition of repressive H2AR3me2s in the genome may occur by a similar mechanism. The presence of pre-existing methylated H2A may serve to potentiate other downstream histone modifications (Loyola et al. 2006), and its introduction into chromatin may directly alter the pre-existing chromatin templates at particular genomic loci.

\section{Concluding remarks}

This study demonstrates a role of Prmt5 function in early mouse development and pluripotency. The nuclear-cytoplasmic translocations of Prmt5 during preimplantation development, PGC specification, and EG and ES derivation coincide with significant developmental transitions and cell fate decisions. We recently generated a conditional allele for Prmt5 to further examine the role of maternal inheritance of Prmt5 in preimplantation embryos and during various stages of development. Notably, the transient loss of Prmt5 in the ICM apparently occurs by post-transcriptional regulation, and may reflect a general mechanism to evoke rapid cell fate decisions. The subsequent up-regulation and cytoplasmic localization of Prmt5 in the emerging ES cells is coupled with the upregulation of Stat3. One purpose of these factors is to repress differentiation. In contrast, up-regulation of cytoplasmic Prmt5 in the post-implantation epiblast cells in vivo occurs without the concomitant up-regulation of Stat3, presumably to allow these embryonic epiblast cells to commence differentiation (Supplemental Fig. 6). Notably, reprogramming of unipotent PGCs to pluripotent EG cells, which also requires LIF/Stat3 signaling, results in the translocation of Prmt5 from the nucleus to the cytoplasm (Durcova-Hills et al. 2008). Given the recent demonstration that Stat3 activation contributes directly to induction of pluripotency (Yang et al. 2010), the functional association of Prmt5 and Stat 3 may provide further insights into the molecular mechanism underlying epigenetic reprogramming toward pluripotency.

In ES cells, Prmt5 acts through Mep50 to mediate methylation of predeposited $\mathrm{H} 2 \mathrm{~A}$ histones, which contributes to the repression of several differentiation genes, some of which are apparently Stat 3 targets. Prmt5 may have additional roles, independent of H2A methylation, in regulating pluripotency, since the response of ES cells to the loss of Mep50 (and H2A ${ }^{\mathrm{R} 3 \mathrm{~A}}$ overexpression) is more specific compared with the more pronounced effect of loss of Prmt5. Indeed, other putative substrates of Prmt5 may also be involved in maintaining ES cell pluripotency; these may include either nonhistones or perhaps other H2A variants. Interestingly, Prmt5 has the potential to methylate all $\mathrm{H} 2 \mathrm{~A}$ variants, with the exception of H2A.Z, which lacks an Arg 3 residue in the N-terminal tail and therefore is refractory to methylation (Supplemental Fig. 7). As such, an interchange between H2A.Z and H2AR3me2s depositions could be important for transcriptional regulation in ES cells. The abundance of predeposited methylated H2A histones in ES cells is reminiscent of that reported in Xenopus oocytes (Shechter et al. 2009). This may be one general property of the totipotent and pluripotent uncommitted states to ensure developmental plasticity.

The cytoplasmic localization of Prmt5 provides a unique experimental system to investigate how epigenetic regulation may be coupled to signal transduction prior to changes in the chromatin template. The reported evidence for cytosolic Ezh2 complex may similarly link additional signal transduction pathways (Su et al. 2005). Our work suggests that Prmt5 may cooperate with the LIF/Stat3 pathway to repress differentiation, and such coupling of signal-induced epigenetic response can provide a mechanism to promote and maintain pluripotency, while preserving the ability to alter the epigenome rapidly upon exposure to differentiation cues.

\section{Materials and methods}

\section{Analysis of Prmt5 mutants and knockdown in ES cells}

Prmt5 $^{+/-}$gene trap ES cells were obtained from BayGenomics (clone RRA014) and injected into C57BL/6 blastocysts to generate chimeric mice. Isolation, stainings, and in vitro culture of blastocysts was performed as described previously (Maldonado-Saldivia et al. 2007). Wholemount immunostainings on E6.5 embryos were carried out as described (Ohinata et al. 2005). shRNA oligonucleotides were cloned into pSuperpuro (Oligoengine).

\section{Gene expression analysis and cell fractionation}

Microarray gene expression profiling was performed using the Illumina microarray platform (mouse WG6 expression BeadChip). Cell fractionation was carried out as described previously, with minor modifications 
(Nimura et al. 2006; Groth et al. 2007). Full experimental details are provided in the Supplemental Material.

\section{Acknowledgments}

We thank Drs. Andy Bannister and Sophie Polo for critical comments on the manuscript, and W.W.T. is grateful to Drs. F. Tang and K. Hayashi for helpful advice and discussions. We thank Drs. Fiona Watt and Toshio Hirano for the kind gift of the Stat3(Y705F) plasmid, and B. Kidder for primer sequences. W.W.T. is a recipient of the Wellcome Trust $\mathrm{PhD}$ studentship. This work is supported by grants from the Wellcome Trust to M.A.S. and J.S.C.

\section{References}

Ancelin K, Lange UC, Hajkova P, Schneider R, Bannister AJ, Kouzarides T, Surani MA. 2006. Blimp1 associates with Prmt5 and directs histone arginine methylation in mouse germ cells. Nat Cell Biol 8: 623-630.

Bedford MT, Clarke SG. 2009. Protein arginine methylation in mammals: Who, what, and why. Mol Cell 33: 1-13.

Bilodeau S, Kagey MH, Frampton GM, Rahl PB, Young RA. 2009. SetDB1 contributes to repression of genes encoding developmental regulators and maintenance of ES cell state. Genes Dev 23: 2484-2489.

Boyer LA, Plath K, Zeitlinger J, Brambrink T, Medeiros LA, Lee TI, Levine SS, Wernig M, Tajonar A, Ray MK, et al. 2006. Polycomb complexes repress developmental regulators in murine embryonic stem cells. Nature 441: 349-353.

Durcova-Hills G, Tang F, Doody G, Tooze R, Surani MA. 2008. Reprogramming primordial germ cells into pluripotent stem cells. PLoS ONE 3: e3531. doi: 10.1371/journal.pone.0003.531.

Friesen WJ, Wyce A, Paushkin S, Abel L, Rappsilber J, Mann M, Dreyfuss G. 2002. A novel WD repeat protein component of the methylosome binds Sm proteins. J Biol Chem 277: 8243-8247.

Furuno K, Masatsugu T, Sonoda M, Sasazuki T, Yamamoto K. 2006. Association of Polycomb group SUZ12 with WD-repeat protein MEP50 that binds to histone H2A selectively in vitro. Biochem Biophys Res Commun 345: 1051-1058.

Goldberg AD, Banaszynski LA, Noh KM, Lewis PW, Elsaesser SJ, Stadler S, Dewell S, Law M, Guo X, Li X, et al. 2010. Distinct factors control histone variant $\mathrm{H} 3.3$ localization at specific genomic regions. Cell 140: 678-691.

Goodfellow H, Krejci A, Moshkin Y, Verrijzer CP, Karch F, Bray SJ. 2007. Gene-specific targeting of the histone chaperone asf1 to mediate silencing. Dev Cell 13: 593-600.

Groth A, Corpet A, Cook AJ, Roche D, Bartek J, Lukas J, Almouzni G. 2007. Regulation of replication fork progression through histone supply and demand. Science 318: 1928-1931.

Gubitz AK, Mourelatos Z, Abel L, Rappsilber J, Mann M, Dreyfuss G. 2002. Gemin5, a novel WD repeat protein component of the SMN complex that binds Sm proteins. J Biol Chem 277: 5631-5636.

Kidder BL, Yang J, Palmer S. 2008. Stat3 and c-Myc genome-wide promoter occupancy in embryonic stem cells. PLOS ONE 3: e3932. doi: 10.1371/journal.pone.0003932.

Loyola A, Bonaldi T, Roche D, Imhof A, Almouzni G. 2006. PTMs on H3 variants before chromatin assembly potentiate their final epigenetic state. Mol Cell 24: 309-316.

Maldonado-Saldivia J, van den Bergen J, Krouskos M, Gilchrist M, Lee C, Li R, Sinclair AH, Surani MA, Western PS. 2007. Dppa2 and Dppa4 are closely linked SAP motif genes restricted to pluripotent cells and the germ line. Stem Cells 25: 19-28.

Matsuda T, Nakamura T, Nakao K, Arai T, Katsuki M, Heike T, Yokota T. 1999. STAT3 activation is sufficient to maintain an undifferentiated state of mouse embryonic stem cells. EMBO J 18: 4261-4269.

Meister G, Eggert C, Buhler D, Brahms H, Kambach C, Fischer U. 2001. Methylation of Sm proteins by a complex containing PRMT5 and the putative U snRNP assembly factor pICln. Curr Biol 11: 1990-1994.

Mosammaparast N, Ewart CS, Pemberton LF. 2002. A role for nucleosome assembly protein 1 in the nuclear transport of histones $\mathrm{H} 2 \mathrm{~A}$ and H2B. EMBO J 21: 6527-6538.

Nimura K, Ishida C, Koriyama H, Hata K, Yamanaka S, Li E, Ura K, Kaneda Y. 2006. Dnmt3a2 targets endogenous Dnmt3L to ES cell chromatin and induces regional DNA methylation. Genes Cells 11: $1225-1237$.
Niwa H. 2007. How is pluripotency determined and maintained? Development 134: 635-646.

Niwa H, Burdon T, Chambers I, Smith A. 1998. Self-renewal of pluripotent embryonic stem cells is mediated via activation of STAT3. Genes Dev 12: 2048-2060.

Ohinata Y, Payer B, O'Carroll D, Ancelin K, Ono Y, Sano M, Barton SC, Obukhanych T, Nussenzweig M, Tarakhovsky A, et al. 2005. Blimp1 is a critical determinant of the germ cell lineage in mice. Nature 436: 207-213.

Pollack BP, Kotenko SV, He W, Izotova LS, Barnoski BL, Pestka S. 1999. The human homologue of the yeast proteins Skb1 and Hsl7p interacts with Jak kinases and contains protein methyltransferase activity. J Biol Chem 274: 31531-31542.

Shechter D, Nicklay JJ, Chitta RK, Shabanowitz J, Hunt DF, Allis CD. 2009. Analysis of histones in Xenopus laevis. I. A distinct index of enriched variants and modifications exists in each cell type and is remodeled during developmental transitions. J Biol Chem 284: 10641074.

Su IH, Dobenecker MW, Dickinson E, Oser M, Basavaraj A, Marqueron R, Viale A, Reinberg D, Wulfing C, Tarakhovsky A. 2005. Polycomb group protein ezh2 controls actin polymerization and cell signaling. Cell 121: 425-436.

Surani MA, Hayashi K, Hajkova P. 2007. Genetic and epigenetic regulators of pluripotency. Cell 128: 747-762.

Takeda K, Noguchi K, Shi W, Tanaka T, Matsumoto M, Yoshida N, Kishimoto T, Akira S. 1997. Targeted disruption of the mouse Stat3 gene leads to early embryonic lethality. Proc Natl Acad Sci 94: 38013804.

Tang F, Barbacioru C, Bao S, Lee C, Nordman E, Wang X, Lao K, Surani MA. 2010. Tracing the derivation of embryonic stem cells from the inner cell mass by single-cell RNA-Seq analysis. Cell Stem Cell 6: 468-478.

Yang J, van Oosten AL, Theunissen TW, Guo G, Silva JC, Smith A. 2010. Stat3 activation is limiting for reprogramming to ground state pluripotency. Cell Stem Cell 7: 319-328.

Yeap LS, Hayashi K, Surani MA. 2009. ERG-associated protein with SET domain (ESET)-Oct4 interaction regulates pluripotency and represses the trophectoderm lineage. Epigenetics Chromatin 2: 12. doi: 10.1186/1756-8935-2-12.

Yuan P, Han J, Guo G, Orlov YL, Huss M, Loh YH, Yaw LP, Robson P, Lim B, Ng HH. 2009. Eset partners with Oct4 to restrict extraembryonic trophoblast lineage potential in embryonic stem cells. Genes Dev 23: $2507-2520$. 


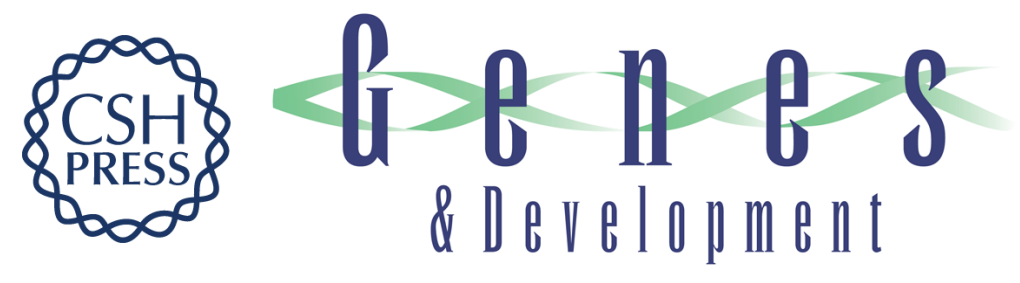

\section{Prmt5 is essential for early mouse development and acts in the cytoplasm to maintain ES cell pluripotency}

Wee-Wei Tee, Mercedes Pardo, Thorold W. Theunissen, et al.

Genes Dev. 2010, 24:

Access the most recent version at doi:10.1101/gad.606110

Supplemental
Material http://genesdev.cshlp.org/content/suppl/2010/12/13/24.24.2772.DC1

References This article cites 30 articles, 12 of which can be accessed free at:

http://genesdev.cshlp.org/content/24/24/2772.full.html\#ref-list-1

License

Email Alerting Receive free email alerts when new articles cite this article - sign up in the box at the top

Service

right corner of the article or click here.

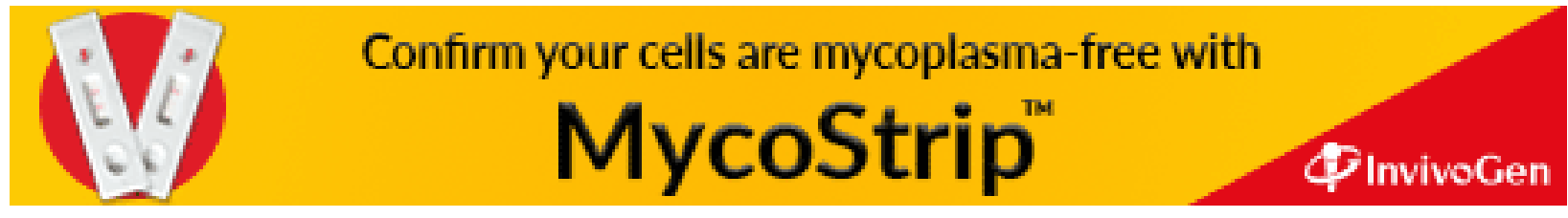

